\title{
Supraconductivité. Introduction
}

Cet ouvrage, labellisé par Grenoble Sciences, est un des titres du secteur Sciences de la matière de la Collection Grenoble Sciences (EDP Sciences), qui regroupe des projets originaux et de qualité. Cette collection est dirigée par Jean BORNAREL, professeur à l'université Joseph FOURIER, Grenoble 1.

Comité de lecture de l'ouvrage :

- Jean Pascal BRISON, chercheur au CEA, service de physique statistique, magnétisme, supraconductivité - CEA Grenoble

- Hervé CourToIs, professeur à l'université Joseph Fourier, Grenoble 1

- Thierry KLEIN, professeur à l'université Joseph Fourier, Grenoble 1

- Jérome LESUEUR, professeur à l'école supérieure de physique et de chimie industrielles, Paris Tech

- Stéphane PAILHÈs, chargé de recherche au CNRS, laboratoire de physique de la matière condensée et nanostructures, Lyon

- José TEIXEIRA, directeur de recherche au CNRS, laboratoire Léon BRILLOUIN, CEA Saclay

- Pierre Vedrine, ingénieur au CEA, institut de recherche sur les lois fondamentales de l'univers, Saclay

- Georges WAYSAND, directeur de recherche au CNRS, laboratoire souterrain à bas bruit, Rustrel

Cet ouvrage a été suivi par Laura CAPOLO pour la partie scientifique et par Sylvie Bordage et Anne-Laure Passavant du centre technique Grenoble Sciences pour sa réalisation pratique. L'illustration de couverture est l'œuvre d'Alice GIRAUD, d'après : image du réseaux de vortex obtenue en spectroscopie tunnel à balayage dans l'équipe de D. RodITCHEV à l'institut des nanosciences de Paris - UMR 75-88 au CNRS, Université Pierre et Marie CURIE, Paris 6; aimant en lévitation au-dessus d'un supraconducteur, J. BobrofF, J. QuILliaM, F. BOUQueT, LPS, Orsay.

\section{Autres ouvrages labellisés sur des thèmes proches (chez le même éditeur)}

Magnétisme : I Fondements, II Matériaux (Sous la direction d'E. du Trémolet de Lacheisserie) • Physique des diélectriques (D. Gignoux \& J.C. Peuzin) • La Mécanique Quantique. Problèmes résolus, Tome I et II (V.M. Galitski, B.M. Karnakov \& V.I. Kogan) • Introduction à la mécanique statistique $($ E. Belorizky \& $W$. Gorecki) • Mécanique Statistique. Exercices et problèmes corrigés (E. Belorizky \& W. Gorecki) - Mécanique - De la formulation lagrangienne au chaos hamiltonien $($ C. Gignoux \& B. Silvestre-Brac) $\bullet$ Problèmes corrigés de mécanique et résumés de cours. De Lagrange à Hamilton $(C$. Gignoux \& B. SilvestreBrac) • Naissance de la Physique (M. Soutif) - L'Asie, source de sciences et de techniques (M. Soutif) - Description de la symétrie. Des groupes de symétrie aux structures fractales (J. Sivardière) - Symétrie et propriétés physiques. Des principes de Curie aux brisures de symétrie (J. Sivardière) - La Turbulence (M. Lesieur) - Turbulence et déterminisme (M. Lesieur en collaboration avec l'institut universitaire de France) $\bullet$ Physique des plasmas collisionnels. Applications aux décharges hautes fréquences (M. Moisan \& J. Pelletier) • Spectroscopie de résonance paramagnétique électronique, fondements ( $P$. Bertrand) Spectroscopies infrarouge et Raman (R. Poilblanc \& F. Crasnier) • Les milieux aérosols et leurs représentations (A. Mailliat)

et d'autres titres sur le site internet :

http://grenoble-sciences.ujf-grenoble.fr 